\title{
Aplicación móvil de control nutricional para prevención de la anemia ferropénica en la mujer gestante
}

\section{(Mobile Application for Nutritional Control and the Prevention of Iron- Deficiency Anemia in Pregnant Women)}

\author{
Joe Llerena-Izquierdo ${ }^{1}$, María Merino-Lazo ${ }^{1}$ \\ ${ }^{1}$ Universidad Politécnica Salesiana, Guayaquil, Ecuador \\ jllerena@ups.edu.ec,mmerinol@est.ups.edu.ec
}

\begin{abstract}
Resumen: Se presenta el desarrollo de una aplicación móvil para el control nutricional de la mujer gestante con riesgo de anemia ferropénica. Diseñado para registrar, consultar y sugerir las cantidades de hierro requeridas. La metodología de investigación es de corte cuantitativo, con enfoque empírico-analítico de nivel descriptivo. Se utiliza la técnica de la encuesta a una población de 200 mujeres en la ciudad de Lima, Perú. El alto índice de anemia en menores de cinco años, bajo peso del bebé al nacer, así como síntomas comunes que padece la mujer en gestación son factores determinantes que pueden mitigarse mediante el control mediado con el uso de la tecnología. Los resultados evidencian que el problema principal está en los registros de control de la alimentación de la mujer gestante muchas veces elaborados de forma tradicional. El $80 \%$ de las participantes indica que el aplicativo tiene un alto potencial en el área médica preventiva como apoyo al proceso de embarazo que evite el padecimiento de la anemia ferropénica considerada un problema de salud en Perú.
\end{abstract}

Palabras clave: Tecnología y medicina, aplicaciones móviles en el sector salud.

\begin{abstract}
This research presents the development of a mobile application to monitor women's nutrition during pregnancy, when they are more at risk of developing irondeficiency anemia. The app allows users to register daily food consumption and uses inputs to generate follow up reports and suggestions. The application allows users to register, consult and suggest required iron quantities according to nutrition guides. The research is quantitative, with a descriptive empirical - analytical focus. A survey is applied to a population of 200 women in Lima, Peru. High levels of children under age five with anemia, low birth weight as well as other common effects derived from anemia during gestation can be mitigated by using technology to improve health monitoring during pregnancy. Results show anemia frequently fails to be identified and treated due to the traditional ways of recording and analyzing a pregnant women's diet. $80 \%$ indicate that the application has great potential when used as a tool for preventive medicine as it supports healthy pregnancies by decreasing cases of iron-deficiency anemia, considered a major health problem in Peru.
\end{abstract}

Keywords: Technology and medicine, health sector mobile applications.

\section{INTRODUCCIÓN}

La anemia que se relaciona con el déficit de hierro es el tipo de entidad que prevalece en pacientes cuando se identifican uno de los tres escenarios (o los tres en el mismo paciente), hierro no disponible, pero se encuentra acumulado, requerimiento de hierro supera a su aporte, y falta de hierro de forma absoluta [1]. Estudios caracterizan a varios factores que atañen a grupos vulnerables en el mundo [2][3][4][5] y entre ellos, uno va relacionado a la malnutrición, que 
mediante un manejo y control médico [6] o por medio de tecnologías que interaccionan con la persona [7][8] puede ser detectado, prevenido y corregido a tiempo.

La organización mundial de la salud determina que un tercio de las mujeres en edad reproductiva tienen deficiencias de hierro y entre las posibles causas es la nutrición, de la cual se puede priorizar para prevenir enfermedades y su mortalidad, mediante la suplementación y fortificación de su alimentación.

Investigaciones previas en Perú, [9][10][11] determinan factores a los que se atribuyen la falta de hierro antes, durante y posterior a la gestación, entre ellos, el factor nutricional es determinante que evite la anemia ferropénica [6][12].

Este trabajo presenta un prototipo de una aplicación móvil para llevar un control nutricional de las mujeres en la etapa de gestación en el medio peruano, que permite tener una guía alimenticia mediante un calendario con sugerencias de alimentos que cumplen con el nivel de hierro determinado por la Organización Mundial de la Salud durante las etapas de gestación de forma que prevenga y se erradique la anemia ferropénica [13].

La aplicación móvil, denominada Mugsa App (mujer gestante sin anemia) se presenta como primer prototipo y se desarrolla para plataformas Android debido al mayoritario uso de equipos, el factor económico y la diversidad de dispositivos móviles con dicho sistema operativo disponibles en la población.

\subsection{Tecnologías al servicio del ser humano}

Cada vez es más imprescindible el uso de las tecnologías que se encuentran al alcance de las personas por medio de canales digitales o sistemas libres de acceso para dispositivos electrónicos. Investigaciones de los autores apuntan sus esfuerzos al uso de las tecnologías emergentes en distintos campos de estudio y en beneficio de sectores de la sociedad como turismo [14], educación [15], salud [8] en contraste con otros autores [7].

La apertura de los profesionales de la salud de trabajar en conjunto con investigadores de otras áreas ha permitido avances en el desarrollo de propuestas [16][17], dando soluciones a las necesidades del ser humano con el fin de enriquecer la calidad del servicio en este campo [18][19].

El uso de las tecnologías, especialmente para la movilidad invade cada vez más en la cotidianidad de las actividades personales y es notable su aceptación cuando se trata de la salud, como por ejemplo aplicativos de control de pasos, pulseras inteligentes para el deporte o para actividades continuas o repetitivas, aplicaciones para un control, registro y prescripción de medicamentos o servicios de asistencia remota para cuidados médicos, externos o telemedicina ingresando a la era de la salud digital, medicina en línea, o derivaciones sociales de comportamiento y cuidado médico aún por investigarse [20][21][22]. Es notable la preocupación por parte de la comunidad médica utilizar nuevos aplicativos tecnológicos que permitan atenuar enfermedades que requieran tratamientos supervisados por componentes desarrollados bajo software, ese es el desafío para nuevas propuestas [23].

\section{MATERIALES Y MÉTODOS}

El presente trabajo de investigación es de corte cuantitativo, desde un enfoque empírico analítico, de nivel descriptivo, dirigido a doscientas mujeres divididas en tres grupos, las que han tenido un solo hijo, las que han tenido más de uno y las que se encuentran en la etapa de gestación, todas ellas residentes en la ciudad de Lima, Perú. Las variables de estudio que se consideran son la tecnología para la salud y la calidad de vida de la mujer en etapa de gestación.

Mugsa App, se desarrolla sobre plataformas Android bajo el entorno de Android Studio y el lenguaje Kotlin. Su diseño funcional se basa en el análisis según las necesidades de la mujer 
gestante como herramienta informativa y guía nutricional con el objetivo de mitigar la anemia ferropénica en la etapa del embarazo.
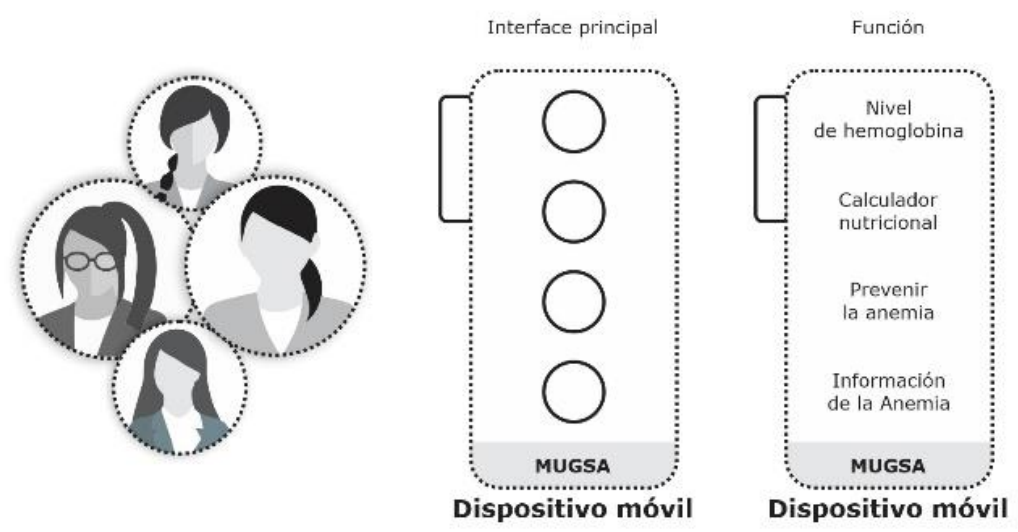

Figura 1. Interface principal de la aplicación Mugsa

La aplicación móvil presenta un menú de cuatro opciones divididos en el módulo del cálculo de hemoglobina, el módulo del calculador nutricional, el módulo informativo: ¿qué es la anemia?, y el módulo informativo: ¿cómo prevenirla? (ver figura 1). El primer módulo permite la consulta del nivel de hemoglobina acorde al tiempo de gestación definido por los estándares mundiales (ver figura 2a). Se muestra el contenido con un enfoque informativo.

Este proceso es realizado las veces que la paciente necesite conocer el nivel adecuado actual. El módulo del calculador nutricional (ver Figura 2b) tiene como propósito presentar el nivel de hierro por alimento seleccionado, segmentados por una lista de categorías de alimentos (ver Figura 2b). La segmentación de categorías y los alimentos con su correspondiente valor de hierro se fundamenta de la tabla de composición de alimentos peruanos [24].

Cuadros textos presentan la cantidad mínima de hierro recomendada por la Organización Mundial de la Salud como la presencia de un sumador automático al seleccionar cada alimento. La información que se presenta es representada por un informe gráfico (ver Figura 2c) mediante porcentajes, con el objetivo de informar al paciente si los alimentos escogidos completan el total de hierro mínimo diario. En caso de no ser el suficiente hierro adquirido, se muestra un conjunto de sugerencias de alimentos para completar la cantidad mínima requerida (ver Figura 2d).

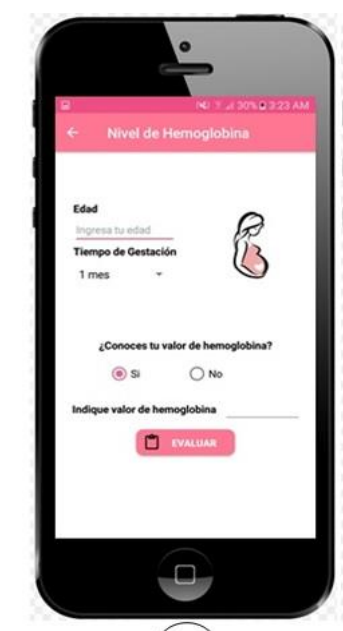

(a)

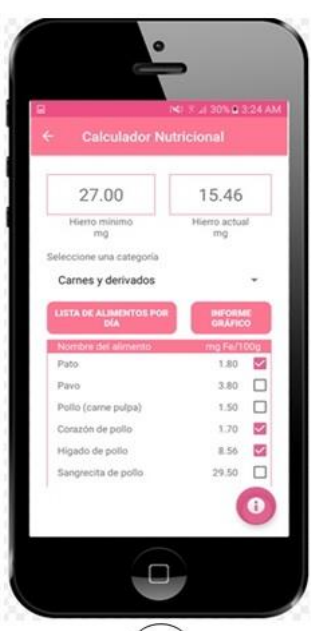

(b)

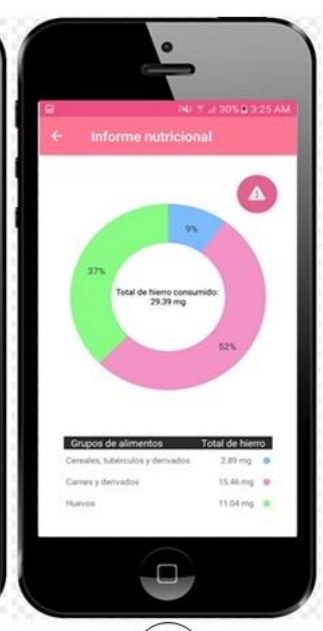

(c)

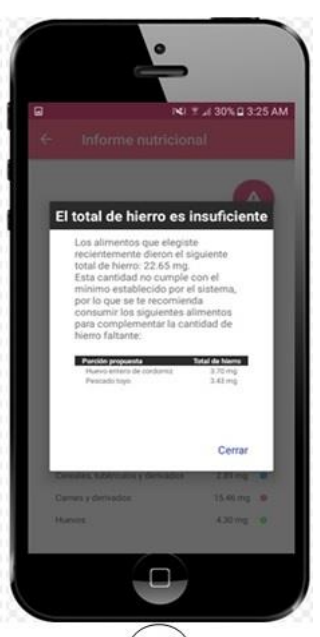

(d)

Figura 2. Interfaces de los módulos de la aplicación Mugsa App. 
El listado de alimentos por día es una opción que permite al calculador nutricional visualizar una pantalla de listados programados para los próximos siete días según el calendario, siguiendo una relación lógica de alimentos seleccionados por cada categoría del calculador nutricional. La aplicación Mugsa App en este primer prototipo, permite al usuario la descarga de un reporte semanal en un formato portable (pdf) como una guía alimenticia por los próximos siete días.

\section{RESULTADOS Y DISCUSIÓN}

De los resultados obtenidos, observamos que el $46 \%$ de las participantes ha tenido un hijo, el $39 \%$ ha tenido más de un hijo, el 10\% se encuentra en la etapa de gestión (cuando se desarrolló la investigación, y un $6 \%$ no ha tenido (ver figura 3).

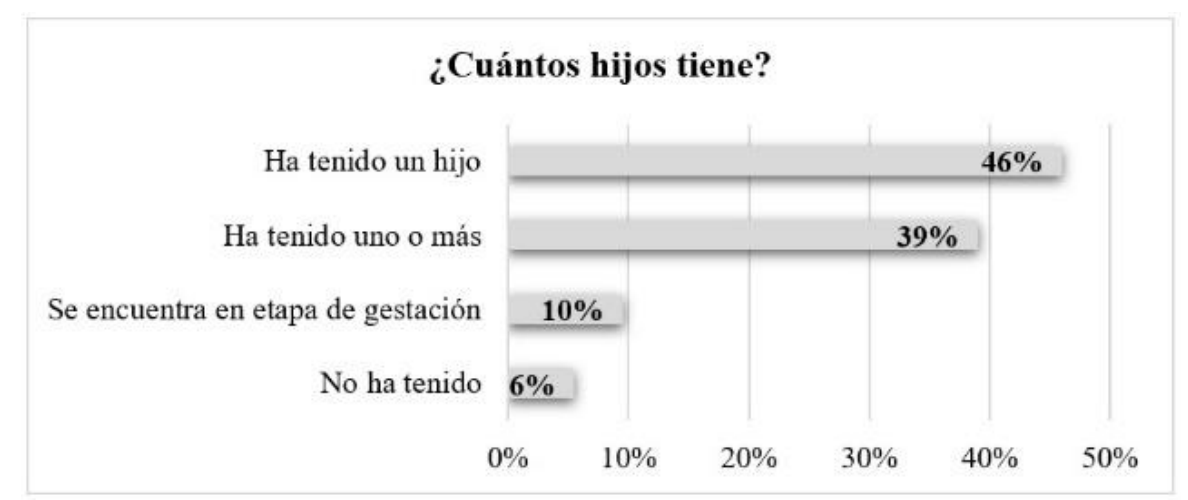

Figura 3. Porcentaje de participantes que han tenido uno o más hijos, las que se encuentran en etapa de gestión y las que todavía no han tenido.

Las edades de las participantes se han clasificado en cuatro categorías, con un $64 \%$ aquellas participantes mayores e igual a 29 años, un $20 \%$ las que se encuentran entre 24 y 28 años, un $15 \%$ aquellas que sus edades están entre 19 a 23 años, y un $2 \%$ menor e igual a 18 (ver figura 4).

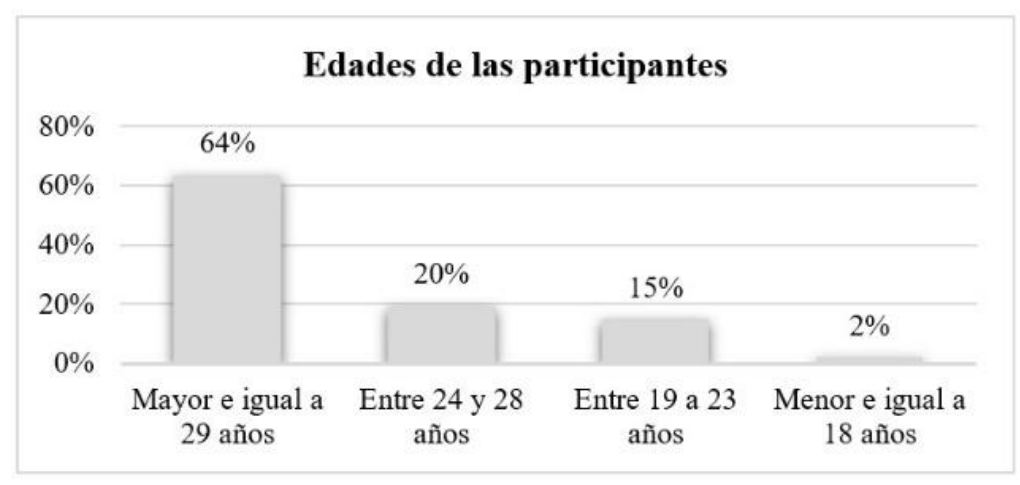

Figura 4. Porcentaje de participantes por edades

Se preguntó al grupo de participantes si conocen en cuántas etapas se agrupa el proceso de gestación de la mujer, respondiendo el $67 \%$ que sí conoce, y el 33\% que no conoce (ver figura $5)$. 


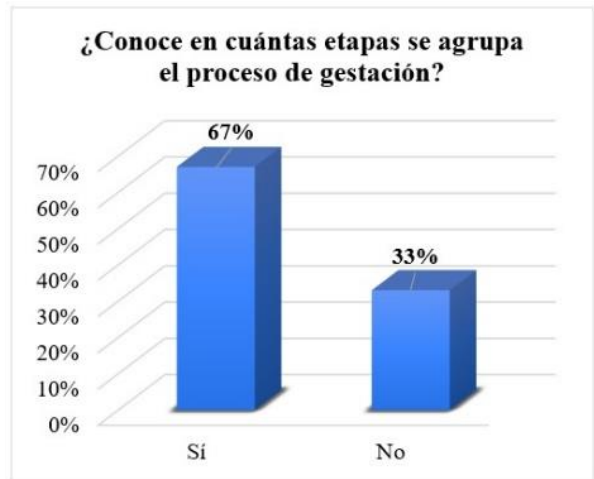

Figura 5. Porcentaje de participantes que conoce y no conoce las etapas del proceso de gestación

Se preguntó al grupo de participantes sobre si conoce el tipo de anemia más frecuente que se da durante el proceso de gestación, obteniendo un $48 \%$ conocen que es frecuente la anemia ferropénica, un $33 \%$ conocen que es frecuente la anemia gravídica, un $15 \%$ conocen que es frecuente la anemia megaloblástica y un $5 \%$ conocen que es frecuente la deficiencia de folato (ver figura 6).

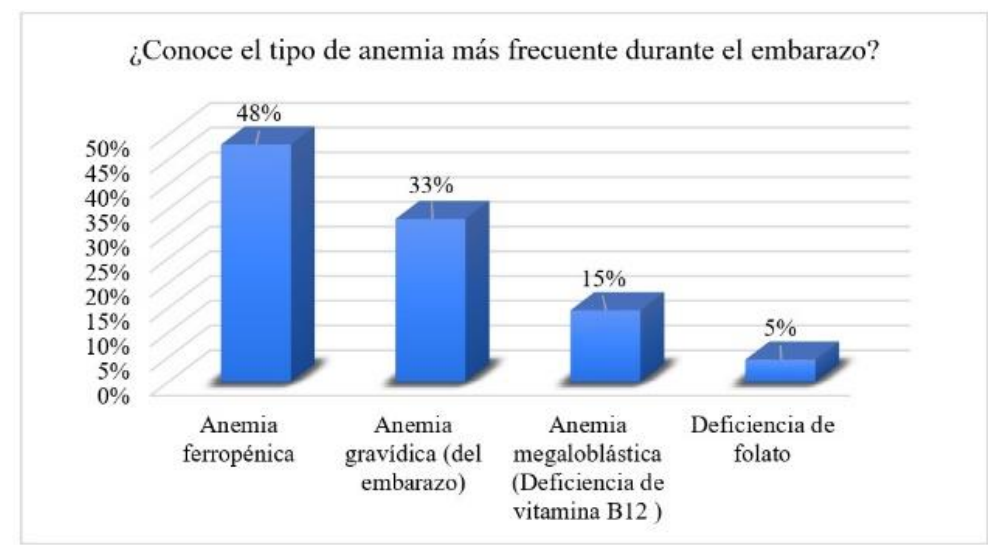

Figura 6. Porcentaje de conocimiento por las participantes, sobre los tipos de anemia

Se observa que es de conocimiento de las participantes de un tipo de anemia que puede afectar a la mujer en la etapa del embarazo.

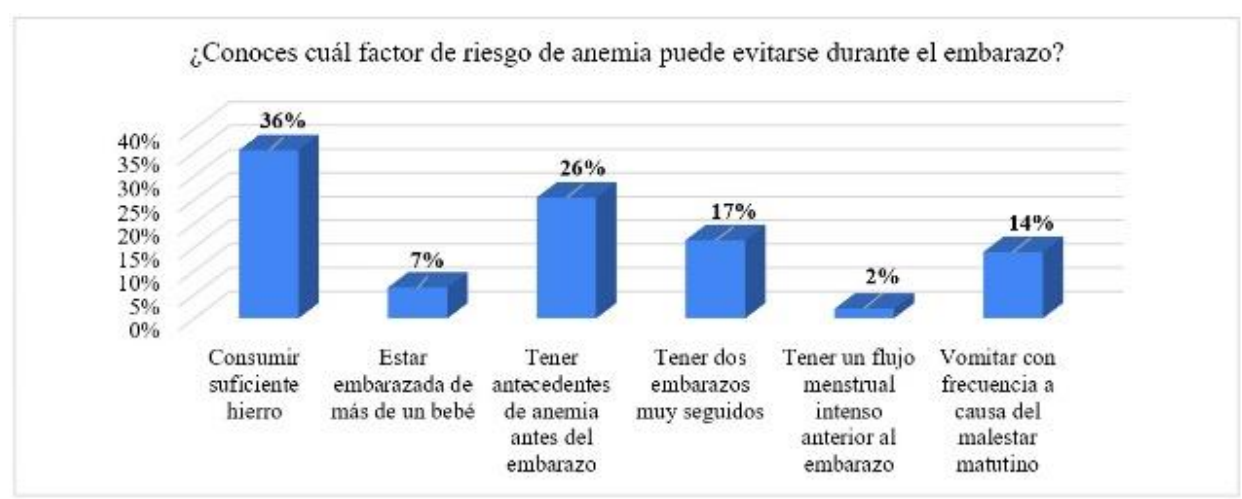

Figura 7. Porcentaje de conocimiento por las participantes, sobre los factores de anemia evitables 
En la figura 7, se muestran los factores de riesgo que conocen las participantes con relación a la anemia, un $36 \%$ indica que la anemia puede prevenirse al consumir una cantidad suficiente de hierro, un $26 \%$ indica que la anemia puede evitarse si se conoce los antecedentes de anemia antes del embarazo, un $17 \%$ indica que la anemia puede evitarse al no tener dos embarazos muy seguidos, un $14 \%$ indica que la anemia puede evitarse al notar vómito con frecuencia a causa del malestar matutino, un 7\% indica que la anemia puede evitarse al conocer que se está embarazada de más de un bebé y el $2 \%$, indica que la anemia puede evitarse al conocer el flujo menstrual intenso anterior al embarazo. Esto demuestra que el conocimiento que tenga la mujer gestante de su propio registro histórico puede conducir a eludir situaciones de riesgo y a la vez prever medidas complementarias ante uno de los factores de riesgo conocido.

Las participantes se les preguntó sobre si conoce cuál complicación es frecuente en las mujeres gestantes entre cuatro opciones propuestas, se obtuvo como resultado que ellas conocen que $74 \%$ de complicaciones durante el embarazo es debido a la anemia por deficiencia de hierro, un $16 \%$ debido a la presión alta, un $6 \%$ debido a un aborto espontáneo y de igual manera un $6 \%$ por hiperémesis gravídica (ver figura 8 ).

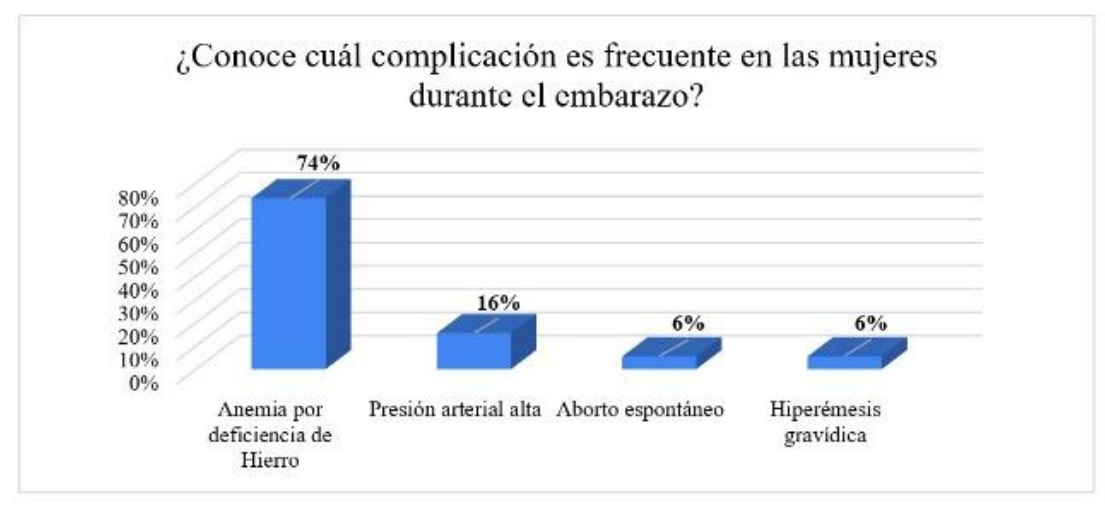

Figura 8. Porcentaje de conocimiento por las participantes, sobre complicaciones frecuentes durante el embarazo

En la figura 9, se observa que el $67 \%$ desconoce la cantidad de hierro mínimo diario para prevenir la anemia, el 20\% indicó de forma incorrecta entre un 20 a $26 \mathrm{mg}$ de hierro, así también un 7\% respondió de forma incorrecta menor a $19 \mathrm{mg}$ de hierro, solo el $6 \%$ indicó que la cantidad de suplemento mínimo para prevenir la anemia debe ser mayor e igual a $27 \mathrm{mg}$. Esto demuestra que el $94 \%$ de la población encuestada ignora el nivel de hierro de ingesta diaria que son importantes para el cuidado de la salud.

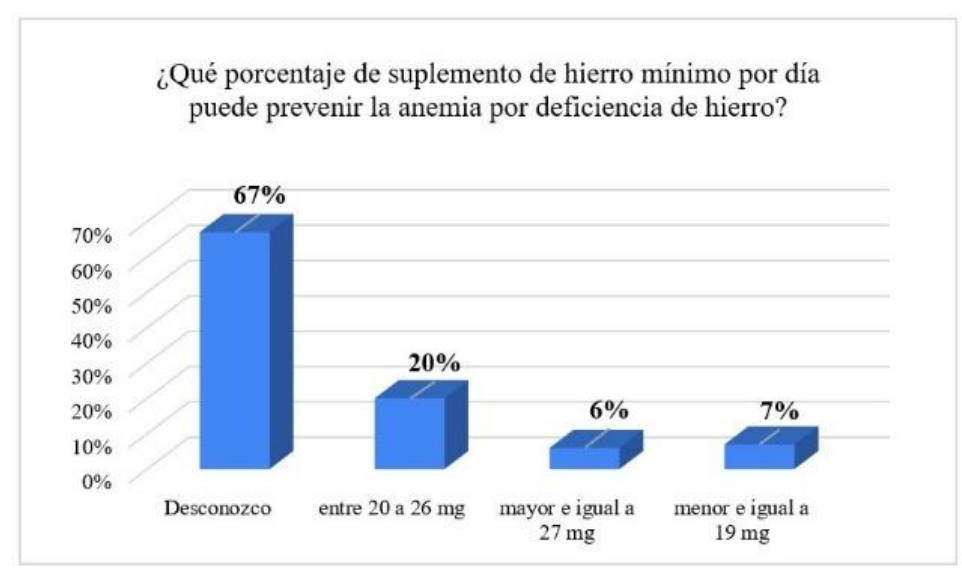

Figura 9. Porcentaje de conocimiento de las participantes de suplemento de hierro en mg. 
En la figura 10, el 33\% indica que Mugsa App puede ayudar en el control nutricional en la mujer gestante en un $100 \%$, el $46 \%$ indica que ayuda en un $75 \%$, un $17 \%$ indica que ayuda sólo en un $50 \%$. Esto demuestra que el $79 \%$ de mujeres encuestadas cree que la aplicación puede ayudar en su cuidado nutricional.

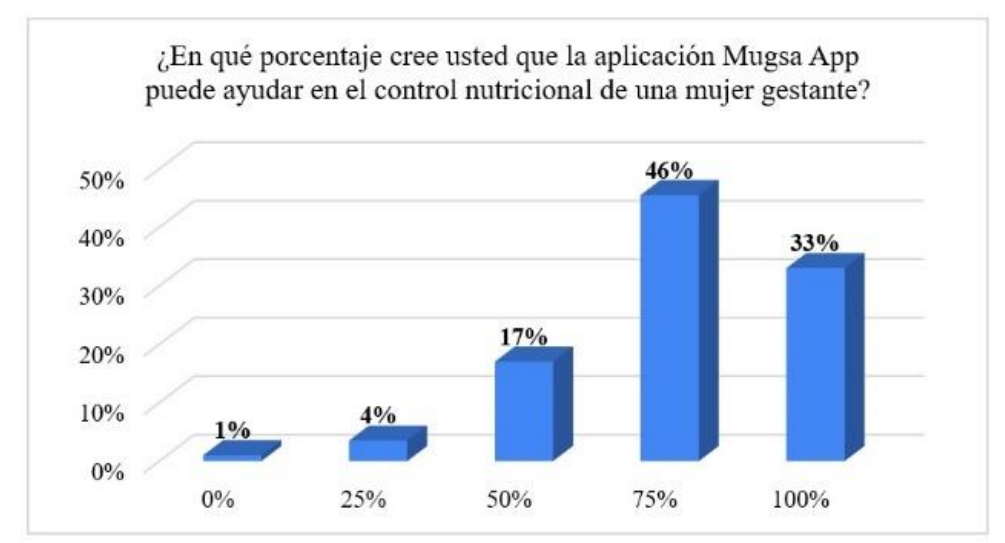

Figura 10. Porcentaje de aceptación de la aplicación Mugsa App

Se preguntó a las participantes sobre el interés en el uso de la aplicación móvil Mugsa App, y se obtuvo que un $37 \%$ está interesado en un $100 \%$, un $42 \%$ está interesado en un $75 \%$ y un $16 \%$ en un 50\%. Podemos entender que un $79 \%$ tiene interés por el uso de la aplicación Mugsa App (ver figura 11).

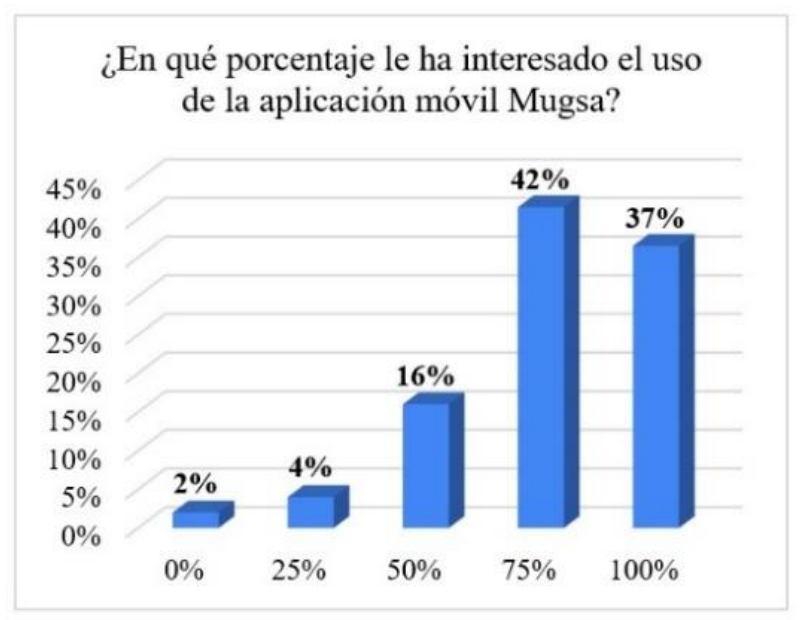

Figura 11. Porcentaje de interés por el uso de la aplicación Mugsa App

Se preguntó a las participantes sobre el interés en el contenido de la aplicación móvil Mugsa App y estos valores aumentan, a un $81 \%$ por el interés, tomando como referencias que el $38 \%$ que está $100 \%$ interesado, con el $43 \%$ de participantes que están interesados en un $75 \%$.

Es resaltable indicar que, como primer prototipo del aplicativo Mugsa App para dispositivos Android, tiene un mayor interés para las participantes el conocimiento que pueden adquirir, que el diseño, línea gráfica y disposición. Se evidencia la motivación por el aplicativo debido al uso de tecnología móvil para la salud, en la forma de prevenir el padecimiento de la anemia en la mujer y en especial en la etapa de gestación (ver figura 12). 


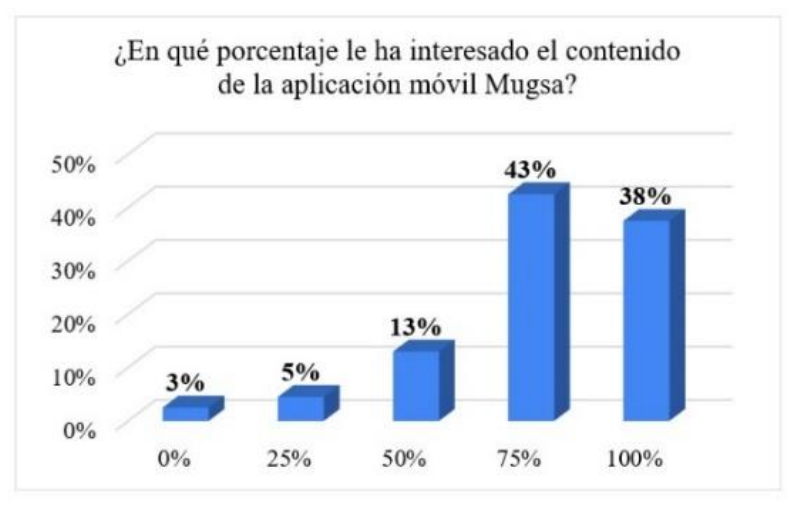

Figura 12. Porcentaje de interés por el contenido de la aplicación móvil Mugsa App

Finalmente se preguntó al conjunto de participantes, si confiaría en el aplicativo Mugsa App para llevar su control nutricional, obteniendo: un 39\% que indican que sí confiarían en 100\%, un $38 \%$ que confiaría en un $75 \%$ y un $19 \%$ que confiaría en un $50 \%$. Con estos resultados, un $77 \%$ confía en Mugsa App para llevar su control nutricional.

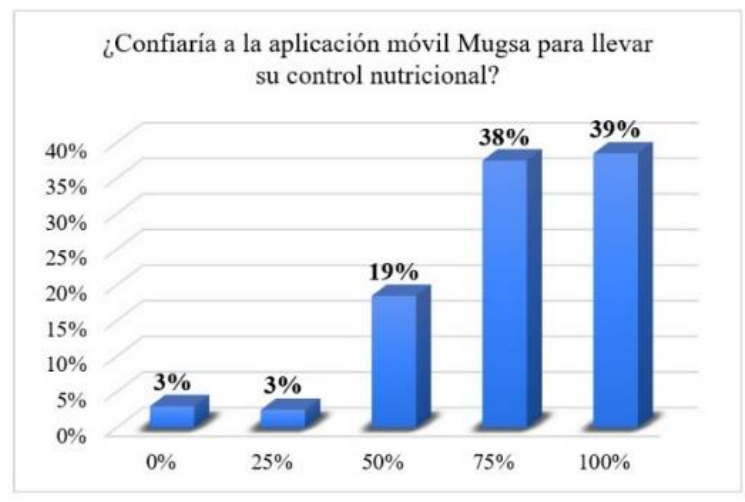

Figura 13. Porcentaje de satisfacción de la aplicación Mugsa App

\section{CONCLUSIONES}

Las aplicaciones móviles son un gran aporte en la actualidad para la vida cotidiana, y en especial manera en los tiempos que vivimos, por su facilidad de acceso, movilidad y disponibilidad. Varias áreas de trabajo, estudio y en salud utilizan cada vez más tecnologías que logren mejorar la calidad de vida de las personas. Este trabajo presenta una propuesta de un aplicativo móvil para un control nutricional que mitigue el padecimiento de la anemia en la mujer gestante. Mugsa App, es una propuesta destinada a mejorar el nivel de conocimiento previo, durante y posterior al parto, con respecto al ámbito alimenticio. Además, sugiere alimentos con su respectivo valor de hierro mediante un calendario alimenticio y además, permite consultar si su estado de salud está acorde al tiempo de gestación.

Finalmente, la anemia ferropénica es un problema frecuente en el Perú donde se evidencia mediante este trabajo que está asociada con problemas de gravedad en la madre como para el recién nacido que, mediante una aplicación con tecnología móvil llega a ser una estrategia de prevención para la anemia, de uso informativo y de guía nutricional.

AGRADECIMIENTOS: Los autores agradecen a la Universidad Politécnica Salesiana y al grupo de investigación en Enseñanza - Aprendizaje de las Ciencias para la Ingeniería, GIECI, por su acompañamiento en el desarrollo de este trabajo. 


\section{REFERENCIAS}

[1] G. Estrada-Garcia, R. Carrillo-Esper, L. Mejía-Gómez, and A. Perez-Calatayud, “Optimización de la Masa Eritrocitaria," J. EMIVA News, vol. 17, no. 3, pp. 16-37, 2020.

[2] R. G. Abdulwahid and H. M. Ahmed, "Nutritional Characteristics of Pregnant Women and its Relation with Anemia during Pregnancy in a Sample of Kurdish Women/Iraq," Cihan Univ. Sci. J., vol. 4, no. 1, pp. 37-44, Jan. 2020, doi: 10.24086/cuesj.v4n1y2020.pp37-44.

[3] M. Ibrahim yousf, ali elshabrawi, S. Ibrahim, and N. Khalifa, "Prevalence Rate and Different Methods of Treatment of Iron Deficiency Anemia Among Pregnant Women Attending Diarb Negm Central Hospital.," Zagazig Univ. Med. J., vol. 0, no. 0, pp. 0-0, Jan. 2020, doi: 10.21608/zumj.2020.18182.1586.

[4] S. A. Leonard, E. K. Main, D. J. Lyell, A. J. Butwick, and S. L. Carmichael, "244: Antepartum iron-deficiency anemia: An opportunity to reduce severe maternal morbidity," Am. J. Obstet. Gynecol., vol. 222, no. 1, pp. S168-S169, Jan. 2020, doi: 10.1016/j.ajog.2019.11.260.

[5] E. Safavi, M. Marzban, L. Sadeghmoghaddam, and A. Farhadi, "Iron deficiency anemia in older females: A comparison between community-dwelling individuals and nursing home residents in the Southwest of Iran," Shiraz E Med. J., vol. 21, no. 3, Feb. 2020, doi: 10.5812/semj.92271.

[6] R. T. Means, "Iron deficiency and iron deficiency anemia: Implications and impact in pregnancy, fetal development, and early childhood parameters," Nutrients, vol. 12, no. 2, p. 447, Feb. 2020, doi: 10.3390/nu12020447.

[7] M. Ahmad, M. A. Khan, M. Bibi, Z. Ullah, and S. T. Shah, "Mobile Apps for Human Nutrition: A Review," in Mobile Devices and Smart Gadgets in Medical Sciences, IGI Global, 2020, pp. 121-147.

[8] J. Llerena-Izquierdo, M. Barberan-Vizueta, and J. Chela-Criollo, "Novus spem, 3D printing of upper limb prosthesis and geolocation mobile application," RISTI - Rev. Iber. Sist. e Tecnol. Inf., vol. 2020, no. E33, pp. 127-140, 2020.

[9] A. Muñoz and P. Cristina, "Factores maternos asociados a anemia gestacional en población alto-andina del Hospital Regional de Ayacucho Miguel Ángel Mariscal Llerena durante Julio Diciembre 2019," Universidad Ricardo Palma, 2016. Accessed: 05-Apr$2020 . \quad$ [Online]. Available: http://repositorio.urp.edu.pe/bitstream/handle/urp/453/Loayza_l.pdf?sequence=1\&isAllo wed $=\mathrm{y}$.

[10] E. Espinoza Portilla, M. Henríquez Suárez, and G. J. Villanueva Cadenas, Oportunidades de aplicación de las tecnologías de la información y comunicación (TICs) para fortalecer la lucha contra la anemia en Perú, vol. 36, no. 2. Colegio Médico del Perú, 2019.

[11] J. R. Melchor Salazar, "Factores sociales asociados a la anemia en gestantes que acuden al Hospital Hipólito Unanue de Tacna, 2018," Universidad Nacional Jorge Basadre Grohmann, 2020.

[12] S. Govindappagari and R. Burwick, "Treatment of Iron Deficiency Anemia in Pregnancy with Intravenous versus Oral Iron: Systematic Review and Meta-Analysis," Am. J. Perinatol., vol. 36, no. 04, pp. 366-376, Mar. 2019, doi: 10.1055/s-0038-1668555.

[13] R. D. Narváez Machado, "Factores asociados con la anemia en lactantes de 6 meses del Hospital de Huaycán, Lima, 2019," Universidad Peruana Unión, 2020. 
[14] J. Llerena, M. Andina, and J. Grijalva, "Mobile application to promote the Malecón 2000 tourism using augmented reality and geolocation," in Proceedings - 3rd International Conference on Information Systems and Computer Science, INCISCOS 2018, 2018, vol. 2018-Decem, pp. 213-220, doi: 10.1109/INCISCOS.2018.00038.

[15] J. L. Izquierdo, M. R. Alfonso, M. A. Zambrano, and J. G. Segovia, "Aplicación móvil para fortalecer el aprendizaje de ajedrez en estudiantes de escuela utilizando realidad aumentada y m-learning," Rev. Ibérica Sist. e Tecnol. Informação, no. E22, pp. 120-133, 2019, Accessed: 11-Mar-2020. [Online]. Available: https://search.proquest.com/docview/2317841837/fulltextPDF/B90BB034CFBB42D5PQ/ 1 ? accountid=32861.

[16] W. J. Brown and R. A. W. Tortorella, "Hybrid medical simulation - a systematic literature review," Smart Learn. Environ., vol. 7, no. 1, p. 16, Dec. 2020, doi: 10.1186/s40561-020-00127-6.

[17] V. Tuncay and P. M. A. van Ooijen, "3D printing for heart valve disease: a systematic review," European radiology experimental, vol. 3, no. 1. NLM (Medline), p. 9, 15-Feb2019, doi: 10.1186/s41747-018-0083-0.

[18] R. Ayala Carabajo, "Pedagogical relationship: Max van manen's pedagogy in the sources of educational experience," Rev. Complut. Educ., vol. 29, no. 1, pp. 27-41, 2018, doi: 10.5209/RCED.51925.

[19] J. Swinnen, M. Keupers, J. Soens, M. Lavens, S. Postema, and C. Van Ongeval, "Breast imaging surveillance after curative treatment for primary non-metastasised breast cancer in non-high-risk women: a systematic review," Insights into Imaging, vol. 9, no. 6. Springer Verlag, pp. 961-970, 01-Dec-2018, doi: 10.1007/s13244-018-0667-5.

[20] J. Llerena-Izquierdo, N. Viera-Sanchez, and B. Rodriguez-Moreira, Portable Device and Mobile Application for the Detection of Ultraviolet Radiation in Real Time with a Low Cost Sensor in Arduino, vol. 1193 CCIS. 2020.

[21] A. Olorukooba, L. Amadu, B. Lawal, U. Umar, and A. L.-B. of Abstracts, "Stream: ICT and reducing the global maternal mortality Global Mhealth: Use of mobile technology to prevent Global maternal mortality," Iiteda.Org, Accessed: 12-Mar-2020. [Online]. Available: https://iiteda.org/abostorage/2020/02/Book-of-AbstractsICITED2019.pdf\#page $=$.

[22] C. Wang, Z. Cheng, X.-G. Yue, and M. McAleer, "Risk Management of COVID-19 by Universities in China," J. Risk Financ. Manag., vol. 13, no. 2, p. 36, Feb. 2020, doi: 10.3390/jrfm13020036.

[23] R. Alegría Guerrero, C. Gonzales Medina, and F. Huachín Morales, "El tratamiento de la anemia por deficiencia de hierro durante el embarazo y el puerperio," Rev. Peru. Ginecol. y Obstet., vol. 65, no. 4, pp. 503-509, 2019, doi: 10.31403/rpgo.v65i2220.

[24] Ministerio de Salud del Perú, Tablas Peruanas de Composición de Alimentos. Instituto Nacional de Salud, 2009. 